\title{
Daily dosimetric quality control of the MM50 Racetrack Microtron using an electronic portal imaging device
}

\author{
M.L.P. Dirkx, M. Kroonwijk, J.C.J. de Boer, B.J.M. Heijmen \\ Dr. Daniel den Hoed Cancer Center, Department of Clinical Physics, Groene Hilledijk 301, 3075 EA Rotterdam, The Netherlands
}

Received 31 January 1995; revision received 17 May 1995; accepted 6 June 1995

\begin{abstract}
The MM50 Racetrack Microtron, suited for advanced three-dimensional conformal radiotherapy techniques, is a complex machine in various respects. Therefore, for a number of gantry angles, daily quality control of the absolute output and fluence profiles of the scanned beams are mandatory. For the applied photon beams, a fast method for these daily checks, based on dosimetric measurements with the Philips SRI-100 Electronic Portal Imaging Device (EPID), has been developed and tested. Open beams are checked for four different gantry angles; for gantry angle 0 , a wedged field is checked as well. Performing and analyzing the measurements takes about $10 \mathrm{~min}$. The applied EPID has favourable characteristics for dosimetric quality control measurements: absolute output measurements reproduce within $0.5 \%$ (I SD) and the reproducibility of relative (2D) beam profile measurements is $0.2 \%$ (1 SD). The day-to-day sensitivity stability over a period of one month is $0.6 \%(1 \mathrm{SD})$. Measured grey scale values are within $0.2 \%$ linear with the applied dose. The $2 \mathrm{D}$ fluence profile of the $25 \mathrm{MV}$ photon beam of the MM50 is very stable in time: during a period of 5 months a maximum fluctuation of $2.2 \%$ has been observed. Once, a deviation in the cGy/MU-value of $6 \%$ was detected. There is no interlock in the MM50-system that would have prevented patient treatment with this strongly deviating output. Based on the results of this study and on clinical requirements regarding acceptability of deviations of beam characteristics, a protocol has been developed including action levels for additional investigations and, if necessary, adjustment of the beam characteristics.
\end{abstract}

Keywords: Dosimetric quality control; Scanning beam; Racetrack microtron; Portal imaging

\section{Introduction}

In the Dr. Daniel den Hoed Cancer Center a dual gantry MM50 Racetrack Microtron $[5,6]$ has been in clinical use since March 1994. This device, built by Scanditronix for the application of advanced threedimensional conformal radiotherapy techniques, produces photon and electron beams with energies from 10 up to $50 \mathrm{M}(\mathrm{e}) \mathrm{V}$ in steps of $5 \mathrm{M}(\mathrm{e}) \mathrm{V}$. Both gantries are equipped with a double focused multileaf collimator (MLC) which is suitable for shaping both photon and electron beams [1]. The MM50 can be used in a fully computer-controlled multi-segment treatment mode [4]. Using this mode only the first segment (field) of a patient treatment needs direct interference of the techni-

\footnotetext{
* Corresponding author.
}

cians; all parameters of the next segments, like the gantry and collimator angles, couch positions, field shape, beam energy and monitor units, are set up by the treatment computer. Flat photon and electron beam profiles are obtained by computer-controlled scanning of elementary beams according to fixed scanning patterns $[2,3]$. In addition, customized scanning patterns can be used to create patient-specific, intensitymodulated beam profiles. Individualized beam profiles can also be produced by means of dynamic multileaf collimation $[7,8]$. Both gantries of the MM50 are equipped with a Philips SRI-100 electronic portal imaging device (EPID) [9].

The MM50 consists of a separate electron accelerator and two gantries. Through an evacuated beam line of about $6 \mathrm{~m}$ the accelerated electrons are transported to one of both gantries where clinical photon and electron 
beams are generated. A constant flow of a helium/nitrogen mixture $\left(99 \% \mathrm{He}, 1 \% \mathrm{~N}_{2}\right)$ runs through the MM50 monitor chambers and the treatment heads to reduce electron scattering, yielding very narrow elementary electron beams [3].

The complex lay-out with a separate accelerator serving two gantries, the application of beam scanning and the continuous gas flow through the monitor chamber and the treatment head definitely require a daily dosimetric quality control program in addition to our more extensive checks on a 2-weekly basis. Therefore, a method has been developed to perform fast dosimetric checks under different gantry angles using the Philips SRI-100 electronic portal imaging device. Measurements take place every morning before patient treatment starts.

This paper deals with a description of the daily quality control measurements and demonstrates the suitability of the EPID for this purpose. The stability of the absolute output and the relative fluence profile of the $25 \mathrm{MV}$ scanned photon beam of the MM50 have been assessed. A protocol for quality control measurements, based on the results of this study, is described.

\section{Materials and methods}

\subsection{The electronic portal imaging device}

The SRI-100 is a fluoroscopic system: radiation incident on a fluorescent screen (at a distance of $160 \mathrm{~cm}$ from the focus) is converted into an optical image which is digitized using a CCD-camera. The CCD-chip consists of $512 \times 256$ pixels, with a pixel size projected at isocenter ${ }^{1}$ of about $0.5 \times 0.8 \mathrm{~mm}^{2}$, resulting in a maximum field size of $25 \times 19 \mathrm{~cm}^{2}$ for imaging. In order to improve the signal-to-noise ratio, the signals of the CCD-camera are accumulated on the CCD-chip and averaged in a frame grabber. The final image is stored in a PC using grey scale values in the range of $0-255$. The technical details of the EPID have been described by Visser et al. [9].

Prerequisites for dosimetric quality control measurements with an EPID are adequate reproducibility, stability and linearity of the measured grey scale values. To ensure the response of the EPID, defined as the ratio of a measured grey scale value and the applied portal dose, to be as unequivocal as possible, some precautions have been taken. The gain and offset of the CCDcamera have been set to fixed values and a fixed data acquisition time has been used. In addition, the images have only been corrected for dark current, which is measured just before irradiation starts.

\footnotetext{
${ }^{1}$ All pixel and field sizes mentioned in this paper are defined at isocenter level, i.e., at a distance of $100 \mathrm{~cm}$ from the focus.
}

The fluorescent screen of the EPID is a $1.65 \mathrm{~mm}$ thick stainless steel plate coated with a fluorescent layer [9]. For a $25 \mathrm{MV}$ photon beam this screen is not thick enough to establish a full charged particle build-up. In the present study this is not a problem since the EPID has only been used for dosimetric constancy checks.

A fixed field size of $18 \times 18 \mathrm{~cm}^{2}$ has been used for all measurements in this study. For data reduction, average grey scale values have been calculated for 49 areas of $15 \times 15 \mathrm{~mm}^{2}$ inside the measured EPID-image, yielding dosimetric data for the beam axis and for 48 off-axis points (see Fig. 1). All software used for acquiring and analyzing the images has been written in the MCL-command language of the SRI-100 system.

In addition to the daily dosimetric checks with the EPID for the $18 \times 18 \mathrm{~cm}^{2}$ field, the field flatness and symmetry are also checked for the maximum field size $\left(31 \times 40 \mathrm{~cm}^{2}\right)$ as part of our 2-weekly quality control program. These measurements are performed along the two main axes using a linear detector array consisting of 32 ionisation chambers.

\subsection{Reproducibility, stability and linearity of the EPID}

The reproducibility, stability and linearity of the EPID have been assessed by comparing measured grey scale values with absolute dose measurements at the beam axis. The dose measurements were performed with an NE-2571 ionisation chamber in a PMMA phantom at the depth of maximum dose with a source-to-surface distance of $100 \mathrm{~cm}$. Since EPID and ionisation chamber measurements could not be performed simultaneously, the two types of measurements were always performed sequentially, keeping the interval time as short as possible.

\subsection{Daily quality control measurements}

For the open $25 \mathrm{MV}$ scanning photon beam, EPID images were obtained on a daily basis for four different gantry angles $\left(0,90,180\right.$ and $\left.270^{\circ}\right)$. For gantry angle 0 , a $60^{\circ}$ wedged field image was measured as well to verify the wedge position and to check the beam penetrative quality. All images were acquired using a fixed dose of 150 Monitor Units. The five fields (four open fields and one wedged field) were set up under computer control using the MM50 multi-segment treatment mode which is described in Section 1.

To determine deviations in the absolute beam output and in the relative $2 \mathrm{D}$ fluence profile, each image has been compared with a corresponding reference image. This reference image reflects the absolute 2D fluence profile at the time of beam commissioning for a fixed dose of $150 \mathrm{MU}$ and a cGy/MU-value of 1 for the $10 x$ $10 \mathrm{~cm}^{2}$ field. Both the absolute output and the 2D fluence profile of the $25 \mathrm{MV}$ photon beam of the MM50 


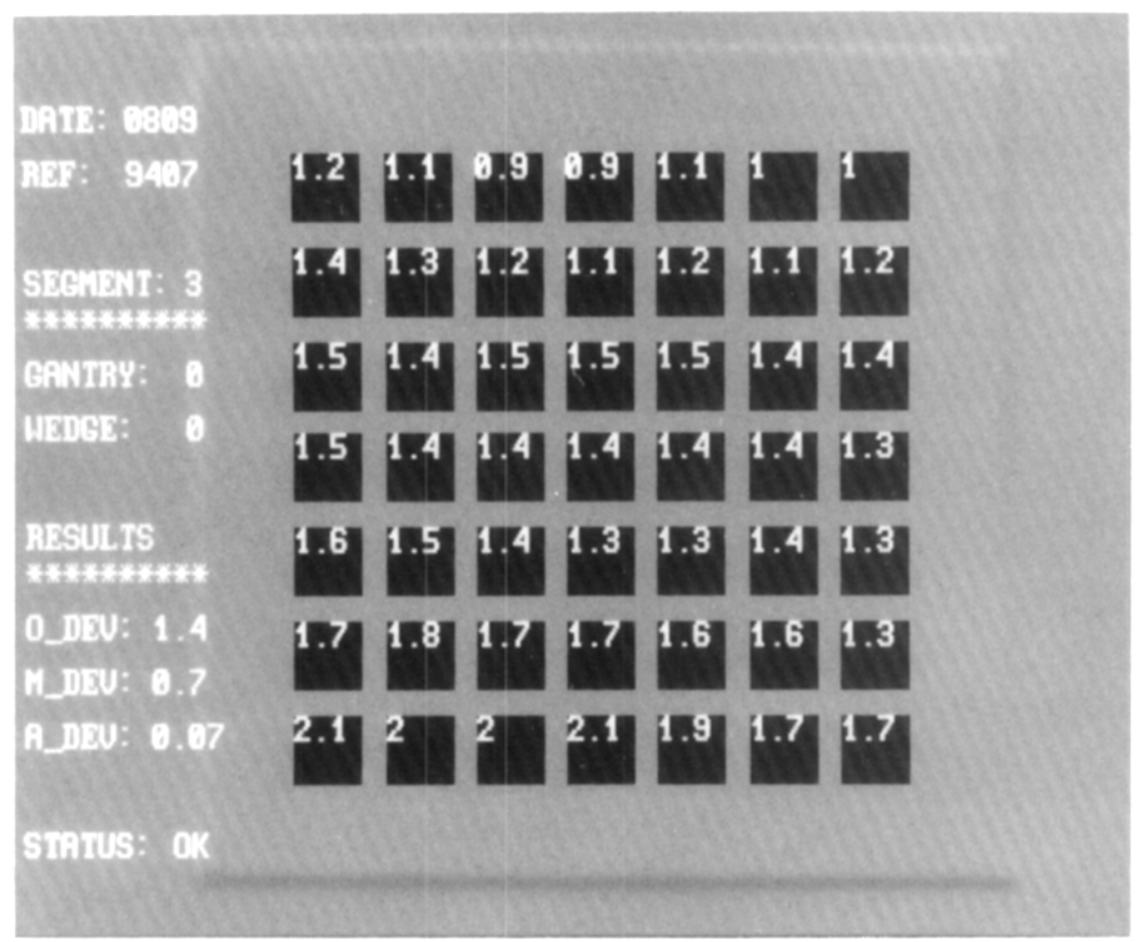

Fig. 1. Screen output showing the definition of the 49 areas of $15 \times 15 \mathrm{~mm}^{2}$ in an EPID-image. The numbers represent an example of observed percentual deviations of measured absolute grey scale values from the corresponding values in the related reference image.

are slightly gantry angle dependent. For clinical practice these dependencies are within acceptable levels and for treatment planning the beam data for gantry angle 0 are used. The use of a separate reference image for each field in the daily dosimetric constancy checks eases a fast interpretation of these measurements: for each field, constancy means zero deviations with regard to its corresponding reference image.

For each of the 49 areas defined in the measured images, the percentual difference in measured grey scale value compared to the corresponding area in the related reference image has been determined. Fig. 1 shows the screen output of a typical measurement. Observed differences at the beam axis ( $1.4 \%$ in this example) relate to deviations in the absolute output of the photon beam. Deviations in the relative 2D fluence profile have been determined by correcting the 49 percentual differences for the observed deviation in absolute output at the beam axis (compare Figs. 1 and 2).

\section{Results and discussion}

\subsection{Reproducibility, stability and linearity of the EPID}

The short-term reproducibility of dosimetric EPIDmeasurements has been assessed by sequential measurement of images and ionisation chamber readings at the beam axis under identical conditions in a short overall period of time (typically $15 \mathrm{~min}$ ). Both for the on-axis and off-axis points the EPID-measurements show a reproducibility of the measured grey scale values of $0.5 \%$ (1 SD); the fluctuations in the ionisation chamber readings were negligible. A significant correlation exists between on-axis and off-axis fluctuations: after correcting the grey scale values in the off-axis points for the observed on-axis fluctuations, the remaining image-toimage variation for off-axis points decreases to only $0.2 \%$ (1 SD).

To assess the day-to-day sensitivity stability of the SRI-100, its response at the beam axis has been determined, usually twice a day, for a period of 20 days. Measurements of one EPID-image and a few ionisation chamber readings were performed for gantry angle 0 in a short overall period of time. The results depicted in Fig. 3 reveal a variation in the on-axis EPID-response of $0.6 \%$ ( $1 \mathrm{SD}$ ), which is only slightly larger than the above-mentioned short-term reproducibility.

To assess the long-term sensitivity stability of the SRI-100, the response measurements have been repeated in a second period of 20 days. The time interval between the first and the second period was 60 days. Also for this second period a variation in the EPID-response of $0.6 \%$ (1 SD) was measured. However, the average response in the second period was $0.5 \%$ lower than in the first period, possibly due to radiation damage in the CCD-chip.

The linearity of the EPID has been assessed for doses between 40 and 180 Monitor Units. From the results in 


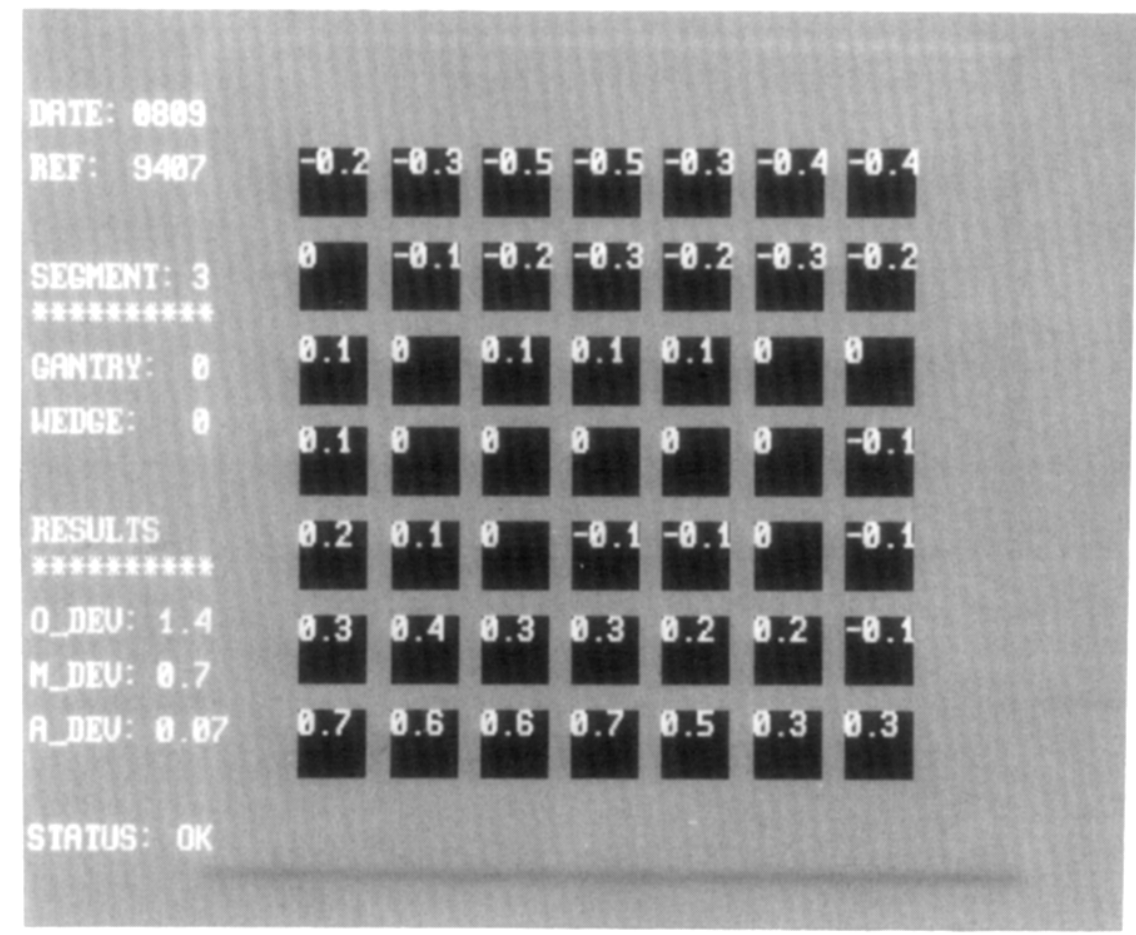

Fig. 2. Percentual deviations in the relative 2D fluence profile from the reference image, derived by renormalization of the data in Fig. 1, assuming zero deviation at the beam axis.

Fig. 4, showing the measured response at the beam axis as a function of MU, it is clear that the response does not significantly depend on the applied dose. In other words, the measured grey scale value increases linearly with the delivered dose.

It may be concluded that the SRI-100 is suitable for daily dosimetric quality control measurements. No adverse interference effects related to the combined use of the MM50 scanning beams and the slow scan mode of the CCD-camera have been observed. For at least a period of several weeks it is possible to derive the output at the beam axis from a single EPID-measurement with an accuracy of $0.6 \%(1 \mathrm{SD})$, while the relative 2D

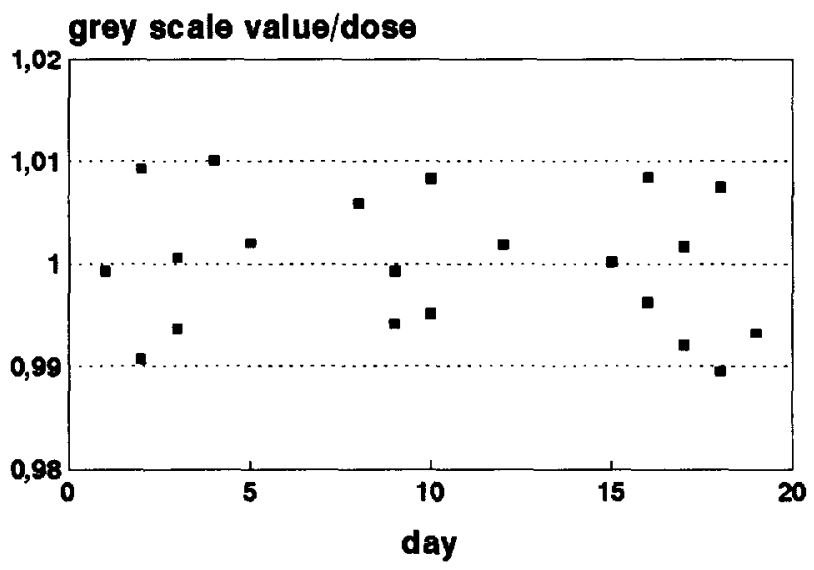

Fig. 3. Day-to-day on-axis sensitivity stability of the EPID. fluence profile can be determined even more accurately. After a period of several weeks a recalibration of the EPID might be necessary to correct for changes in sensitivity.

\subsection{Daily quality control measurements}

The observed deviations in absolute on-axis output for gantry angle 0, measured with the EPID during a period of nearly 150 days, are depicted in Fig. 5. The sudden changes in output deviation on days 38 and 114 are related to recalibrations of the MM50 monitor

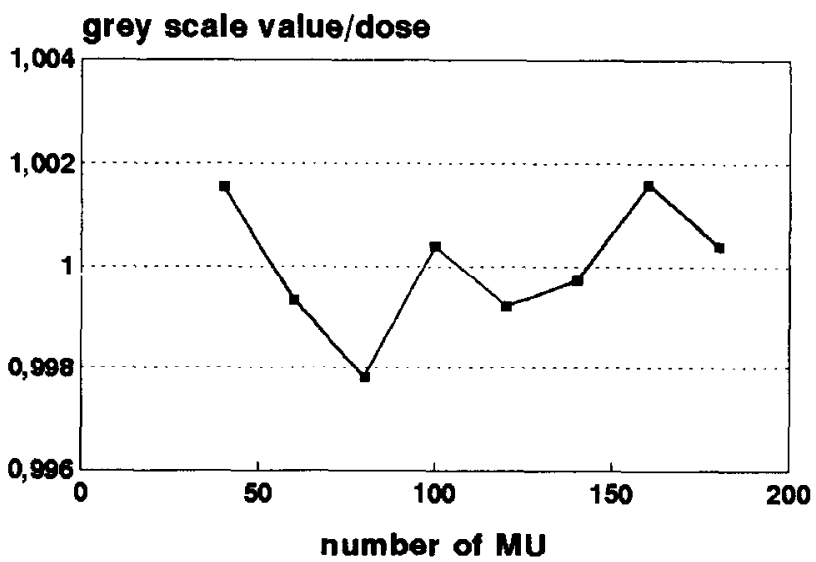

Fig. 4. Linearity of the measured grey scale value with applied portal dose. 
on-axis output deviations (\%)

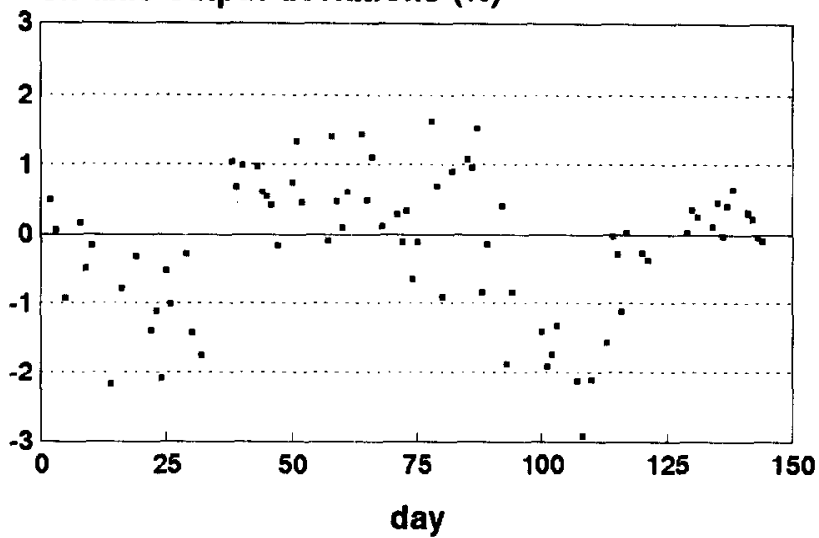

Fig. 5. Deviations in absolute on-axis output of the $25 \mathrm{MV}$ photon beam, measured with the EPID for gantry angle 0 . The deviation of $6 \%$, detected on day 105, has been omitted (see text for further explanation).

chamber. Table 1 summarizes the results of 400 measured images, equally spread over the five different fields.

On day 105 an output deviation of about $6 \%$ was detected for each field. (In order to stick to an appropriate ordinate scale in Fig. 5, this value has not been included.) Checks with an ionisation chamber showed an output deviation of the same amount. This large deviation was a consequence of a human error related to the helium/nitrogen flow in the monitor chamber. For historical reasons two separate bottles are used to supply each gantry with this mixture: one while the MM50system is switched on, the other when the system is off. During the weekend the 'system-off bottle' for one gantry became empty because the person responsible forgot to change it before the weekend. For this reason the constant gas flow stopped, the overpressure in the treatment head went down and air could leak into the treatment

Table 1

Overview of observed deviations from corresponding reference images

\begin{tabular}{llc}
\hline & Deviations (\%) & $\begin{array}{l}\text { Incidence } \\
\text { rate (\%) }\end{array}$ \\
\hline absolute on-axis output & $< \pm 2.0$ & 93 \\
deviations & $\pm(2.0-3.0)$ & 5 \\
& $> \pm 3.0$ & 2 \\
average off-axis profile & $< \pm 0.3$ & 96 \\
deviations & $\pm(0.3-0.5)$ & 4 \\
& $> \pm 0.5$ & 0 \\
maximum off-axis profile & $< \pm 1.5$ & 92.5 \\
deviations & $\pm(1.5-2.0)$ & 7 \\
& $> \pm 2.0$ & 0.5
\end{tabular}

The incidence rates indicate the percentage of the total number of analyzed fields $(n=400)$ in which a certain deviation occurred.

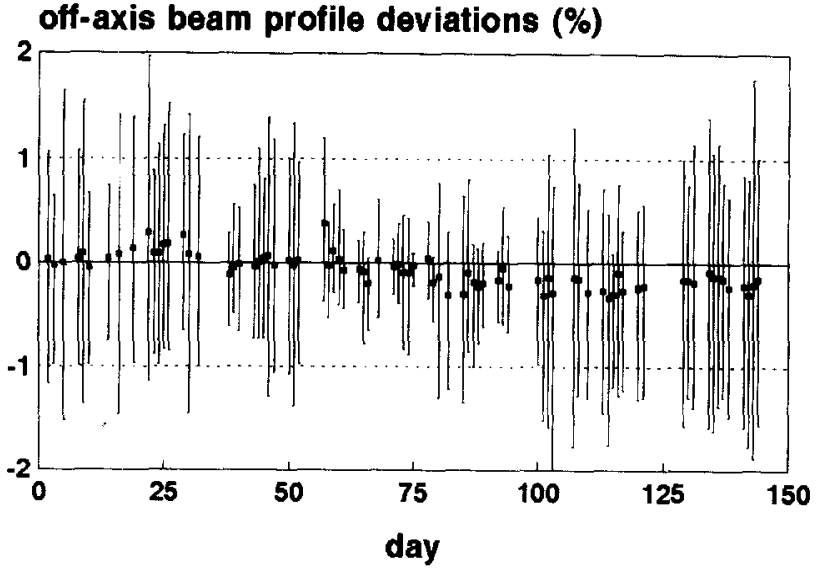

Fig. 6. Off-axis deviations in the relative 2D beam profiles for gantry angle 0 . The dots indicate the average off-axis deviations for the 48 offaxis points, the bars indicate the maximum deviations.

head and therefore also into the monitor chamber. When the machine was switched on after the weekend, the 'system-on bottle' was automatically connected to the monitor chamber. At that moment the MM50system observed correct input and return gas flows (since the 'system-on bottle' was not empty) and no interlock occurred. However, it took several hours before all air had disappeared from the monitor chamber, yielding an increased sensitivity of this chamber and therefore a $6 \%$ too low beam output.

The observed average and maximum deviations in the relative $2 \mathrm{D}$ beam profile, determined from the measurements in the $\mathbf{4 8}$ off-axis points, are depicted in Fig. 6 for gantry angle 0, and summarized in Table 1 for all fields. Because the average off-axis deviation was always less than $0.5 \%$ and the maximum observed offaxis deviation was $2.2 \%$, it may be concluded that the relative 2D fluence profile is very stable.

Wedge factors have been calculated from measured grey scale values at the beam axis for the open and the wedged field at gantry angle 0 . Over the period of this study the measured wedge factor was constant within $0.9 \%$ (1 SD).

On several days, the morning quality control measurements were repeated in the afternoon after patient treatment. No significant differences between results obtained in the morning and in the afternoon have been observed.

\subsection{Protocol for daily quality control measurements}

Based on the results in Table 1 and on clinical requirements regarding acceptability of deviations, the following practical protocol for future daily dosimetric quality control measurements has been adopted: 
If

* for each field the on-axis output deviation is smaller than $3 \%$,

and

* for the five fields the average output deviation at the beam axis is less than $2 \%$ or between 2 and $2.5 \%$ while being below the $2 \%$ level the previous 2 days,

and

* for each field the maximum off-axis beam profile deviation is less than $2 \%$ or between 2 and $2.5 \%$ while being below the $2 \%$ level the previous 2 days,

and

* for each field the average off-axis deviation is less than $0.5 \%$ or between 0.5 and $0.7 \%$ while being below the $0.5 \%$ level the previous 2 days,

the results are accepted and patient treatment can start.

In all other cases, additional investigations and, if necessary, adjustment of the beam characteristics have to be performed. When this protocol had been applied to the daily quality control measurements described before, patient treatment could have started immediately on $95 \%$ of the days.

Daily EPID measurements should be fast. Therefore, only one image is measured per field and the allowed deviations, as described in the protocol above, are somewhat larger than for the checks in our 2-weekly quality control program.

\section{Conclusions}

Because of its characteristics, the Philips SRI-100 Electronic Portal Imaging Device, is suitable for fast and accurate daily dosimetric quality control of photon beams under different gantry angles. Deviations in the absolute output and in the relative 2D fluence profiles are determined within $10 \mathrm{~min}$ for five fields.

Despite the complexity of the MM50, the 2D fluence profile of the $25 \mathrm{MV}$ scanned photon beam has been very stable for all gantry angles during a period of 5 months: a maximum profile deviation of $2.2 \%$ was observed and the average profile deviation never exceeded $0.5 \%$. Once, a deviation in the cGy/MU-value of $6 \%$ was detected. Only because of the performed morning quality control checks, could erroneous dose delivery to patients be avoided. Based on our experiences, a practical protocol for future daily dosimetric quality control measurements has been developed.

This paper is based on the results of measurements for the 25-MV photon beam of one gantry. However, at this moment the dosimetric checks are also successfully performed at the other gantry. In the near future the quality control measurements will be extended to the 10-MV photon beam and geometric checks on multileaf collimator-defined fields will be added as well. Studies on the application of the SRI-100 for in vivo dosimetry on the MM50 have been started.

\section{Acknowledgments}

The authors want to thank Dr. H. Huizenga for his comments on the manuscript and N. Driver and D. Binnekamp for performing the daily quality control measurements. This work was supported by grant No. DDHK 94-848 awarded by the Dutch Cancer Society.

\section{References}

[1] Karlsson, M. and Zackrisson, B. Matching of electron and photon beams with a multileaf collimator. Radiother. Oncol. 29: 317-326, 1993.

[2] Karlsson, M., Nyström, H. and Svensson, H. Photon beam characteristics of the MM50 Racetrack Microtron and a new approach for beam quality determination. Med. Phys. 20: 143-149, 1993.

[3] Karlsson, M., Nyström, H. and Svensson, H. Electron beam characteristics of the 50-MeV Racetrack Microtron. Med. Phys. 19: 307-315, 1992.

[4] Mageras, G.S., Fuks, Z., O'Brien, J., Brewster, L.J., Burman, C., Chui, C.S., Leibel, S.A., Ling, C.C., Masterson, M.E., Mohan, R. and Kutcher, G.J. Initial clinical experience with computer-controlled conformal radiotherapy of the prostate using a $50 \mathrm{MeV}$ Medical Microtron. Int. J. Radiat. Oncol. Biol. Phys. 30: 971-978, 1994.

[5] Masterson, M.E., Mageras G.S., M.E., LoSasso, T., Jöreskog, E., Larsson, L.G., Mohan, R., Ling, C.C., Fuks, Z. and Kutcher, G.J. Preclinical evaluation of the reliability of a 50 MeV Racetrack Microtron. Int. J. Radiat. Oncol. Biol. Phys. 28: 1219-1227, 1994.

[6] Rosander, S., Sedlacek, M. and Wernholm, O. The $50 \mathrm{MV}$ Racetrack Microtron at the Royal Institute of Technology Stockholm. Nucl. Instrum. Methods 204: 1-20, 1982.

[7] Spirou, S.V. and Chui, C.S. Generation of arbitrary intensity profiles by dynamic jaws or multileaf collimators. Med. Phys. 21: 1031-1041, 1994.

[8] Svensson, R., Källman, P. and Brahme, A. An analytical solution for the dynamic control of multileaf collimators. Phys. Med. Biol. 39: 37-61, 1994.

[9] Visser, A.G., Huizenga, H., Althof, V.G.M. and Swanenburg, B.N. Performance of a prototype fluoroscopic radiotherapy imaging system. Int. J. Radiat. Oncol. Biol. Phys. 18: 41-50, 1990. 